\title{
Effects of changing dietary fat content on plasma gut hormone concentrations in diet-induced obese and diet-resistant rats
}

\author{
Jie Li, Shuran Wang*, Na Zhang, Ze Li, Rui Li and Cong Li \\ Department of Nutrition and Food Hygiene, School of Public Health, Harbin Medical University, Harbin, Heilongjiang, \\ People's Republic of China
}

(Received 25 January 2010 - Revised 4 September 2010 - Accepted 28 September 2010 - First published online 9 November 2010)

\section{Abstract}

Gut hormones play key roles in the regulation of energy homeostasis. However, little is known about the long- and short-term effects of changing dietary fat content on gut hormones. We aim to examine the effects of changing dietary fat content on plasma gut hormone concentrations in diet-induced obese (DIO) and diet-resistant (DR) rats. After inducing obesity with a high-fat (HF) diet, male Sprague-Dawley rats were divided into three groups according to their body-weight gain: DIO; DR; control (CON). The DIO and DR rats were further divided in random into two groups. One continued on a HF diet and the other switched to a low-fat (LF) diet for an additional 4 weeks. Finally, each group was randomly divided into three subgroups ( $n$ 8): fasted; fasted-refed HF; fasted-refed LF diet groups. Replacing a HF diet with a LF diet for 4 weeks resulted in less fat mass, higher fasting and post-HF plasma ghrelin concentration and lower postprandial plasma cholecystokinin concentration in the DIO and DR rats. Acute switching dietary fat resulted in significantly higher post-HF plasma ghrelin concentrations than post-LF ghrelin concentrations in the DR rats on LF diet (DRLF) and DIO rats on LF diet (DIOLF) rats, and significantly higher post-HF obestatin concentrations than post-LF obestatin concentrations in the CON, DR rats on HF diet (DRHF) and DRLF rats. Dietary fat content appears to play a role in the gut hormone profile, which may consequently influence fat mass.

Key words: Gut hormones: Dietary fat content: Diet-induced obesity

The gastrointestinal tract is a primary endocrine organ. More than twenty different regulatory peptide hormones are secreted by the gut. These gut hormones play key roles in appetite regulation and energy homeostasis ${ }^{(1)}$. Ghrelin is so far the only orexigenic hormone. Ghrelin is released from the stomach during fasting and might signal directly to the hypothalamus or through the vagus nerve to stimulate food intake. Ghrelin exists in two distinct isoforms: acetylated and desacetylated ghrelin, both of which are derived from the same precursor preproghrelin. Besides regulating short-term appetite, ghrelin participates in long-term energy homeostasis. For instance, chronic administration of ghrelin in rodents results in prolonged hyperphagia and weight gain ${ }^{(2,3)}$. In vitro, ghrelin appears to mediate fat deposition, with the lipogenic effects in part mediated by the $\mathrm{Y} 1$ receptor ${ }^{(4)}$. Obestatin is a peptide encoded by the ghrelin gene, whose physiological function remains obscure ${ }^{(5-8)}$.
Other appetite-regulating peptides from the gut, such as glucagon-like peptide-1 (GLP-1 $)^{(9)}$, peptide tyrosinetyrosine $(\mathrm{PYY})^{(10,11)}$ and cholecystokinin $(\mathrm{CCK})^{(12)}$, characteristically relay a sense of 'fullness' resulting in postprandial satiation and meal termination. They are released into the circulation after a meal. GLP-1 is a potent incretin - central or peripheral administration strongly stimulates insulin release. It exists in several forms, but the most common circulating form is GLP-1 $1_{7-36}$. PYY occurs in two forms: $\mathrm{PYY}_{1-36}$ and $\mathrm{PYY}_{3-36}$. Most circulating PYY immunoreactivity is in the amino-terminally truncated form, $\mathrm{PYY}_{3-36}$. The chronic administration of $\mathrm{PYY}_{3-36}$ results in weight loss in obese rodents ${ }^{(13,14)}$. Transgenic mice overexpressing PYY are protected against diet-induced obesity (DIO) and genetic obesity ${ }^{(15)}$. PYY $3-36$ and GLP-1 can directly stimulate anorectic pathways in the hypothalamus and brainstem, and may also act through the vagus nerve. The arcuate nucleus is important in integrating the gut hormone energy homeostasis signals. Neuropeptide Y/agouti-related

Abbreviations: CCK, cholecystokinin; CON, control; DIO, diet-induced obesity; DIOHF, diet-induced obese rats on a high-fat diet; DIOLF, diet-induced obese rats on a low-fat diet; DR, diet resistant; DRHF, diet-resistant rats on a high-fat diet; DRLF, diet-resistant rats on a low-fat diet; GLP-1, glucagonlike peptide 1; HF, high fat; LF, low fat; PYY, peptide tyrosine-tyrosine; WAT, white adipose tissue.

*Corresponding author: S. Wang, fax +8645187502885, email shuranwang@163.com 
peptide neurons and pro-opiomelanocortin neurons signal to the paraventricular and other hypothalamic nuclei to increase or decrease appetite, respectively ${ }^{(1)}$.

Levin et al. ${ }^{(16,17)}$ discovered that when Sprague-Dawley rats are fed a high-fat (HF) diet, some rats develop obesity, while others remain lean. This phenomenon has also been confirmed in our laboratory ${ }^{(18)}$. Since the diet-induced obese (DIO) rat model closely resembles the polygenic nature of human obesity ${ }^{(19,20)}$, it has been extensively used in obesity research ${ }^{(16,17,21)}$. During the obesity induction phase, rats have free access to either a HF and highenergy diet or a control (CON) diet (low-fat (LF) and low-energy). After the DIO and diet-resistant (DR) phenotypes are established, the effects of switching from a HF diet back to a LF diet become an interesting topic of study. Levin et al. ${ }^{(22)}$ found that when switched to a LF diet, the DR rats eat 13\% less, gain 55\% less weight and have $49 \%$ lower food efficiency, whereas the DIO rats eat $4 \%$ less but have comparable weight gain and efficiency with the CON rats. Huang et al. ${ }^{(23)}$ learned that the replacement of a HF diet with a LF diet is associated with a lowered fasting plasma total PYY concentration in DIO mice, which are changed to a LF diet. Moreover, Aziz et al. ${ }^{(24)}$ found that modifying the starch type in the diet can affect some gut hormones. However, to our knowledge, no study has been carried out to systematically examine the effects of changing dietary fat content on gut hormones. Thus, we aimed to examine the long- and short-term effects of changing dietary fat content on some plasma gut hormone concentrations in the DIO and DR rats.

\section{Methods}

\section{Animals and diets}

All experimental procedures were approved by the Animal Ethics Committee of Harbin Medical University and conducted in compliance with the guidelines for animal use.

Adult male Sprague-Dawley rats ( $n$ 144; body weight 170-190 g) were purchased from the Vital River Laboratories (Beijing, China). They were housed individually in wire cages with controlled environmental conditions (temperature $22 \pm 1^{\circ} \mathrm{C}$, relative humidity $60 \%$, light cycle from 08.00 to 20.00 hours). The rats were fed standard laboratory chow (Keaoxieli, Beijing, China) for the first week to adapt to a new environment. In the following experimental period, the rats were given either a modified control AIN-93G diet (LF diet, $16.4 \mathrm{~kJ} / \mathrm{g}$ ) or a HF diet $(22.0 \mathrm{~kJ} / \mathrm{g}$ ) (Table 1). All micronutrients, proteins and fibre in the HF and LF diets were balanced in terms of energy. The only variation was the relative energy contribution of fats and carbohydrates, with 12 and $60 \%$ from fat. Equal parts of the insoluble fibre (cellulose) and soluble fibre (inulin) were provided to closely resemble the natural ingredients in the diet. The HF and LF diet formulations, which were made from semi-synthetic materials, were in the powder form.
Table 1. Composition of the diets*

\begin{tabular}{lcc}
\hline Diet component & LF diet† $(\mathrm{g} / \mathrm{kg})$ & HF diet $(\mathrm{g} / \mathrm{kg})$ \\
\hline Maize starch & 330 & 130 \\
Dextrin & 165 & 65 \\
Sucrose & 165 & 65 \\
Soyabean & 48 & 64 \\
Lard & 4 & 283 \\
Casein (vitamin free) & 200 & 270 \\
L-Cystine & 3 & 4 \\
Inulin & 20 & 27 \\
Powdered cellulose & 20 & 27 \\
Mineral mix§ & 35 & 48 \\
Vitamin mix§ & 10 & 14 \\
Choline bitartrate & 2 & $2 \cdot 7$ \\
Energy density (kJ/g diet) & $16 \cdot 4$ & $22 \cdot 0$ \\
\hline
\end{tabular}

LF, low fat; HF, high fat.

* In the diets, all micronutrients, proteins and fibre were balanced by energy, the only variation was the relative energy contribution of fats and carbohydrates, with 12 and $60 \% \mathrm{~kJ}$ from fat.

† Containing $11.9 \%$ fat, $67.3 \%$ carbohydrates and $20.8 \%$ protein by energy. ₹ Containing $59.4 \%$ fat, $19.8 \%$ carbohydrates and $20.8 \%$ protein by energy. $\S$ AIN-93G mineral and vitamin mixes ${ }^{(38)}$.

\section{Experimental protocol}

The experiment comprises three phases: HF DIO phase; dietary intervention phase; refeeding phase (Fig. 1).

HF DIO phase (weeks 0-6). After the acclimatisation period, the rats were placed on a HF diet. After 2 weeks, rats with intermediate weight gains ( $n$ 24) were switched to a LF diet and designated as controls, while the other rats were continued on a $\mathrm{HF}$ diet. At the end of week 6, the rats with higher weight gains were designated as DIO rats ( $n$ 48), the rats with lower weight gains were designated as DR rats ( $n$ 48) and the remaining rats were excluded from the study.

Dietary intervention phase (long-term effects, weeks 7-10). After 6 weeks, the DIO and DR rats were further divided in random into two groups each ( $n$ 24). One group continued on a HF diet (DIO rats on HF diet (DIOHF) and DR rats on HF diet (DRHF), while the other was switched to a LF diet (DIO rats on LF diet (DIOLF) and DR rats on LF diet (DRLF) for 4 weeks. The CON rats continued on a LF diet.

Refeeding phase (short-term effects, last day of the experiment). After 10 weeks, each group was randomly divided into three subgroups ( $n$ 8): fasted; fasted-refed $\mathrm{HF}$; fasted-refed LF groups. In the fasted group, the rats were deprived of food for $13 \mathrm{~h}$ (19.00-08.00 hours) with free access to water. In the fasted-refed group, rats in each subgroup were deprived of food for $12 \mathrm{~h}$ (19.0007.00 hours) and refed the HF/LF diet (200 kJ) for $1 \mathrm{~h}$ (07.00-08.00 hours), respectively.

\section{Energy intake and body weight}

The $24 \mathrm{~h}$ food intake was measured every day. Food was weighed at 09.00 hours, and the remaining food and spillage were collected and weighed at the end of a $24 \mathrm{~h}$ period. Energy intake (kJ) was determined by multiplying 


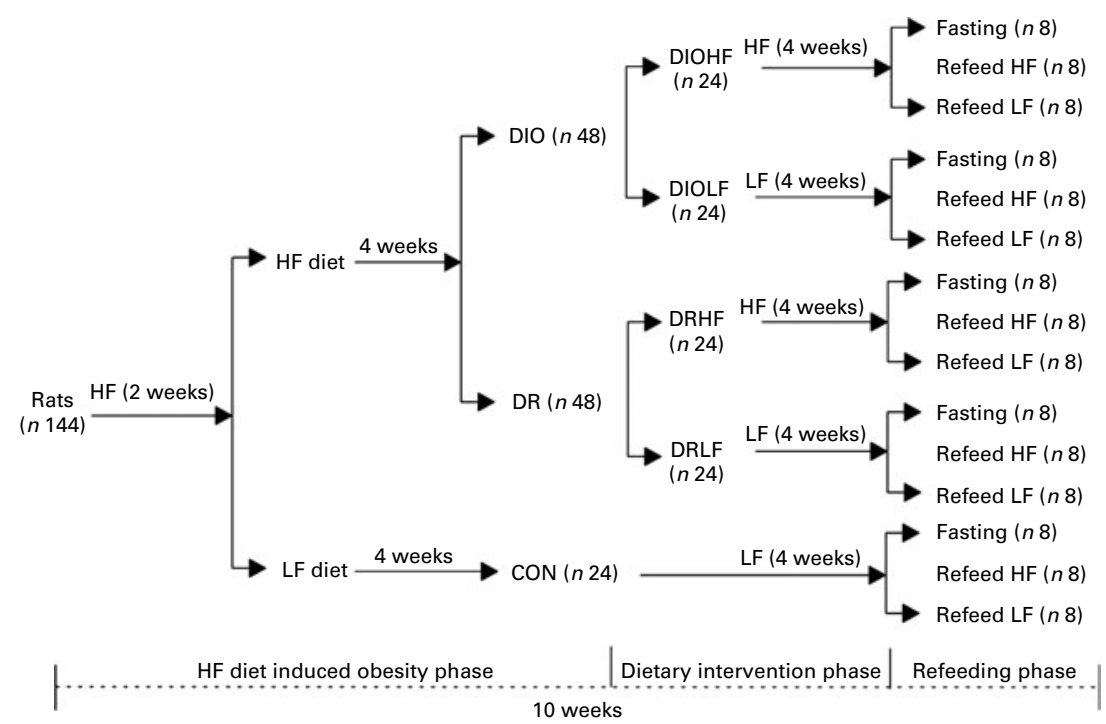

Fig. 1. Three phases of the study: high-fat (HF) diet-induced obesity phase; dietary intervention phase; fasted-refed phase. LF, low fat; DIO, diet-induced obesity; DR, diet resistant; CON, control; DIOHF, DIO on HF diet; DIOLF, DIO on LF diet; DRHF, DR on HF diet; DRLF, DR on LF diet.

food weight $(\mathrm{g})$ and energy density $(\mathrm{kJ} / \mathrm{g})$ of each diet together. Body weight $(\mathrm{g})$ was recorded weekly.

\section{Preparation of plasma samples}

Rats were anaesthetised with an intraperitoneal injection of sodium pentobarbital ( $40 \mathrm{mg} / \mathrm{kg}$ per body weight) at 08.00 hours. For the determination of ghrelin, obestatin, CCK, PYY and GLP-1 concentrations, blood samples were collected from the abdominal aorta and immediately transferred into chilled polypropylene tubes containing EDTA-2Na. These tubes were gently rocked several times immediately after blood collection to get an even mixture and to prevent coagulation. Blood from the tubes was transferred to the centrifuge tubes containing 0.6 trypsininhibiting units $(60 \mu \mathrm{l})$ of aprotinin per ml blood (Applichem GmbH, Darmstadt, Germany) and gently rocked for several times to inhibit the activity of proteinases. Blood samples were centrifuged at $1600 \mathrm{~g}$ for $15 \mathrm{~min}$ at $4^{\circ} \mathrm{C}$. Plasma were collected and stored at $-80^{\circ} \mathrm{C}$ until the assay.

\section{Assays of gut hormones}

Plasma total ghrelin concentrations were measured using an ELISA kit (Linco Research, St Charles, MO, USA). The theoretical minimum detection concentration of this assay was $0.02 \mathrm{ng} / \mathrm{ml}$ total ghrelin. The intra- and inter-assay variations reported by the manufacturer were $<5$ and $<14 \%$, respectively. Plasma obestatin, $\mathrm{CCK}_{26-33}, \mathrm{PYY}_{3-36}$ and GLP-1 ${ }_{7-36}$ concentrations were determined using fluorescent ELISA kits (Phoenix Pharmaceuticals, Belmont, CA, USA). The sensitivities of these assays were $38.9 \mathrm{pg} / \mathrm{ml}$ obestatin, $9.5 \mathrm{pg} / \mathrm{ml} \mathrm{CCK}_{26-33}, 10.7 \mathrm{pg} / \mathrm{ml} \mathrm{PYY} \mathrm{PY}_{36}$ and $20 \cdot 8 \mathrm{pg} / \mathrm{ml} \mathrm{GLP}-1_{7-36}$, respectively. The intra-assay variation was $5-10 \%$, and the inter-assay variation was $<15 \%$.

\section{Statistical analysis}

Data were presented as means with their standard errors. All statistical analyses were performed using SPSS 16.0 (SPSS, Inc., Chicago, IL, USA), with $\alpha=0 \cdot 05$.

Weekly body-weight and energy intake data generated from the HF DIO phase were analysed by a two-way repeated-measures ANOVA (phenotype $\times$ time), followed by Tukey's post hoc test. The other data generated from this phase were analysed by a one-way ANOVA, followed by Tukey's post hoc test.

Weekly body-weight and energy intake data generated from the dietary intervention phase were analysed by a three-way repeated-measures ANOVA (phenotype $x$ dietary intervention $\times$ time), followed by Tukey's post boc test. Total cumulative energy intake and white adipose tissue (WAT) data were analysed by a two-way ANOVA (phenotype $\times$ dietary intervention), followed by Tukey's post boc test. The data for plasma ghrelin, obestatin, CCK, PYY and GLP-1 were analysed by a three-way ANOVA (phenotype $\times$ dietary intervention $\times$ refeeding status), followed by Tukey's post hoc test.

The relationships between fasting plasma ghrelin, obestatin, CCK, PYY, GLP-1 concentrations, dietary intervention and body weight were examined using bivariate correlations. Independent variables potentially influencing body weight were tested by a multiple linear regression analysis.

\section{Results}

High-fat diet-induced obesity phase (weeks 0-6)

A two-way repeated-measures ANOVA revealed significant main effects of phenotype $(P<0.0001)$ and time $(P<0 \cdot 0001)$, as well as a significant interaction between phenotype and time $(P<0.0001)$, on body weight and 
energy intake. After 2 weeks on a HF diet, a distinct phenotype of body weight was observed (Fig. 2(a)): the DIO rats had a higher body weight gain than the CON and DR rats $(P<0 \cdot 0001)$. By the end of week 6 , the DIO rats had a higher body weight gain than the DR $(307 \cdot 3$ (SEM 3.7) v. 233.4 (SEM 3.8) g; $P<0.0001$ ) and CON rats (307.3 (sem 3.7) v. 252.1 (sem 4.9) g; $P<0.0001$ ). The DIO rats consumed significantly more food than the DR and CON rats (Fig. 2(b)). The total cumulative energy intake over this 6-week period was higher in the DIO rats than in the DR $(16.9(\operatorname{sem~} 0 \cdot 1) v \cdot 15 \cdot 0(\operatorname{sem~} 0 \cdot 1) \mathrm{MJ} ; P=0 \cdot 001)$ and CON rats (16.9 (SEM $0 \cdot 1) \quad v$. $15.4 \quad(\operatorname{sem} 0 \cdot 2) \mathrm{MJ}$; $P=0 \cdot 001$ ). When the diet of the CON rats was switched from HF to LF, their weekly energy intake decreased by $19.0 \%$. After 2 weeks, the energy intake in the CON rats increased by $14.6 \%$.

\section{Dietary intervention phase (weeks 7-10)}

Body weight, energy intake and white adipose tissue. At the beginning of this phase, half of the DIO and DR rats were switched to a LF diet (DIOLF and DRLF) for 4 more
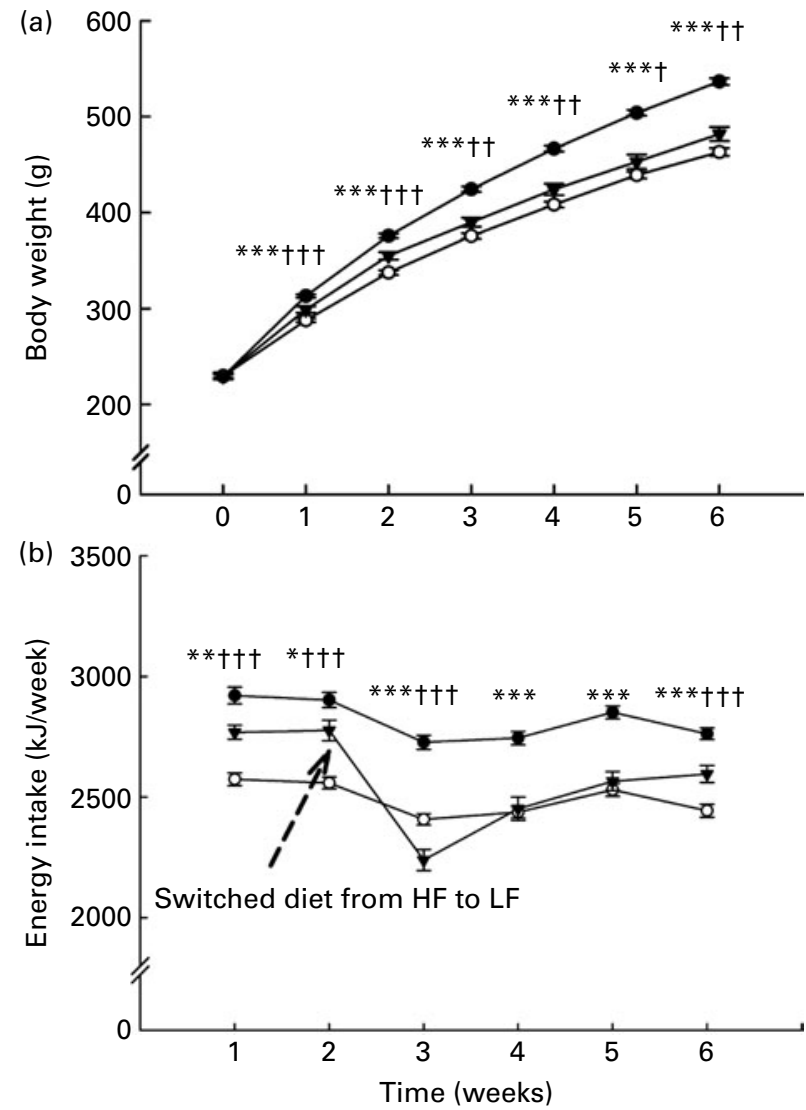

Fig. 2. Body-weight changes (a) and energy intake (b) during the dietinduced obesity phase in rats that were fed a low-fat (LF; control (CON), $n$ 24) or a high-fat (HF) diet (diet-induced obesity (DIO), $n 48$ and dietresistant (DR), $n$ 48). Values are means, with standard errors represented by vertical bars. $\nabla, \mathrm{CON}$; $\bullet$ DIO; O, DR. DIO was different from DR and CON: ${ }^{\star} P<0.05,{ }^{\star \star} P<0.01$ and ${ }^{\star \star \star} P<0.001$, respectively. DR was different from CON: $\dagger P<0.05, \dagger \dagger P<0.01$ and $\dagger \dagger P<0.001$, respectively.
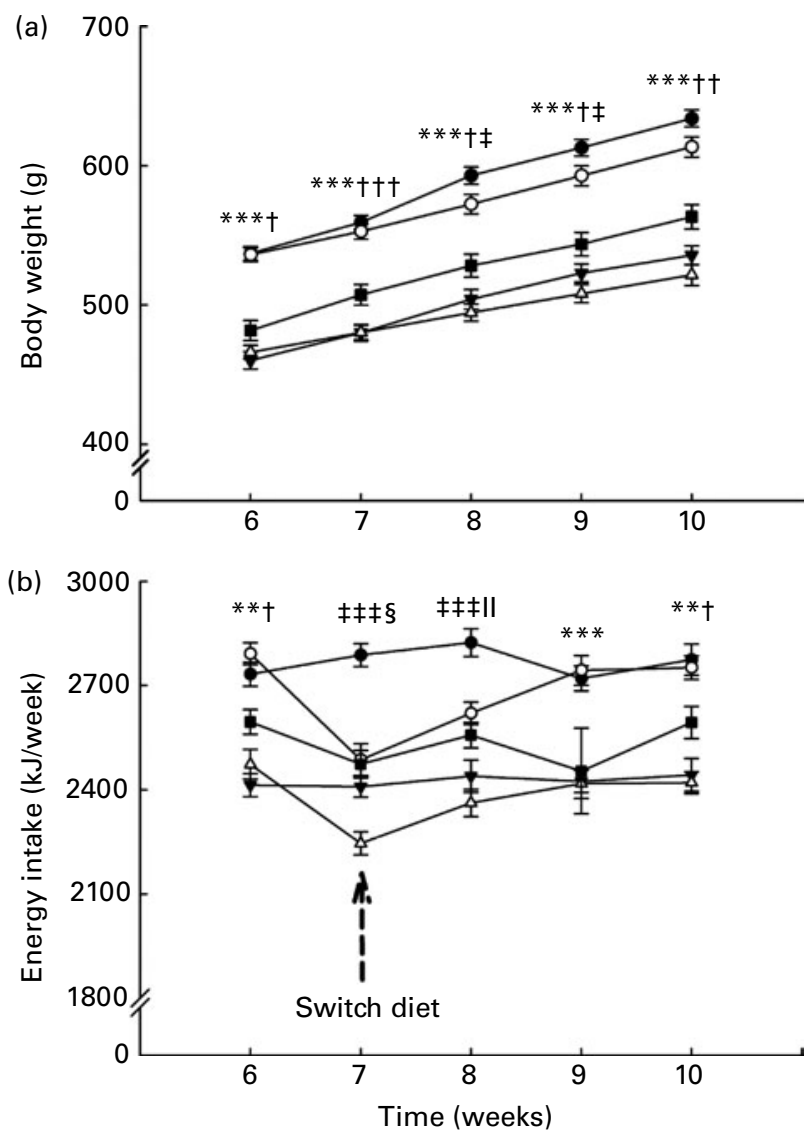

Fig. 3. Body-weight changes (a) and energy intake (b) during the dietary intervention phase in the rats that were fed a low-fat (LF; control (CON, 口), diet-induced obese rats on low-fat diet (DIOLF, O) and diet-resistant rats on low-fat diet (DRLF, $\triangle$ )) or a high-fat diet (diet-induced obese rats on high-fat diet (DIOHF, •) and diet-resistant rats on high-fat diet (DRHF, $\mathbf{\nabla})$ ). Values are means, with standard errors represented by vertical bars, for $n 24$. DIOHF and DIOLF were different from CON, DRHF and DRLF: ${ }^{\star \star} P<0.01$ and ${ }^{\star \star \star} P<0.001$, respectively. DRHF and DRLF were different from CON: $\dagger P<0.05$, $\dagger \dagger P<0.01$ and $\dagger+P<0.001$, respectively. DIOHF was different from DIOLF, CON, DRHF and DRLF: $\ddagger P<0.05$ and $\ddagger \ddagger \ddagger P<0.001$, respectively. DRLF was different from DIOLF, CON and DRHF: $\S P<0.05$. CON and DIOLF were different from DRHF and DRLF: $\| P<0.05$.

weeks, while the other half continued on a HF diet (DIOHF and DRHF). The analysis by a three-way repeated-measures ANOVA revealed significant effects of phenotype $(P<0.0001)$ and time $(P<0.0001)$, as well as interactions between phenotype and time $(P<0.0001)$ and between intervention and time $(P<0.0001)$, on body weight. The DIO rats had a significantly higher body weight than the DR and CON rats over the interventional period. In weeks 8 and 9, the body-weight gain of the DIOLF rats was significantly lower than that of the DIOHF rats. However, this difference disappeared when the weekly energy intake was considered as a covariate. The body-weight gain of the DRLF rats was similar to that of the DRHF rats over the interventional period (Fig. 3(a)).

There were significant effects of time $(P<0.0001)$, phenotype $(P<0.0001)$ and intervention $(P=0 \cdot 001)$, as well as an interaction between time and intervention $(P<0.0001)$, on weekly energy intake. The DIO rats had 
a significantly higher energy intake than the CON and DR rats. The DIOLF and DRLF rats decreased their energy intake by 11 and 9\%, respectively, over the first week on a LF diet. After 2 weeks, their energy intake recovered to the pre-interventional level (Fig. 3(b)).

The effects of phenotype $(P=0.007)$ and dietary intervention $(P=0.047)$ on WAT $(P<0.05)$ were observed. The DIO rats had more WAT than the DR rats (46.3 (SEM 2.7) v. 33.7 (SEm 2.7) g; $P=0.002$ ). Moreover, switching from a HF diet to a LF diet resulted in lower WAT (43.9 (SEM 2.7) v. 36.7 (SEM 2.2) g; $P=0.044$ ). There was no interaction between phenotype and dietary intervention on WAT.

\section{Plasma gut hormone concentrations}

There were main effects of phenotype $(P<0.0001)$, dietary intervention $(P<0.0001)$ and refeeding status $(P<0.0001)$, as well as an interaction between dietary intervention and refeeding status $(P<0 \cdot 0001)$, on plasma ghrelin levels. The DIO rats had significantly lower plasma ghrelin concentrations than the DR $(P<0.0001)$ and CON $(P<0.0001)$ rats. Switching from a HF diet to a LF diet resulted in significantly higher plasma ghrelin concentrations than consuming a HF diet $(P<0 \cdot 0001)$. In addition, post-LF plasma ghrelin concentrations were significantly lower than fasting concentrations $(P<0 \cdot 0001)$ in each group. However, the postprandial plasma ghrelin concentrations were suppressed by a HF diet only in the DRHF and DIOHF rats $(P<0.0001 ;$ Fig. $4(a))$.

There was a main effect of refeeding status $(P<0 \cdot 0001)$ and an interaction between phenotype and refeeding status $(P=0 \cdot 0001)$ on plasma obestatin levels. Post-HF plasma obestatin concentrations significantly increased compared with fasting concentrations in the DRLF, DRHF
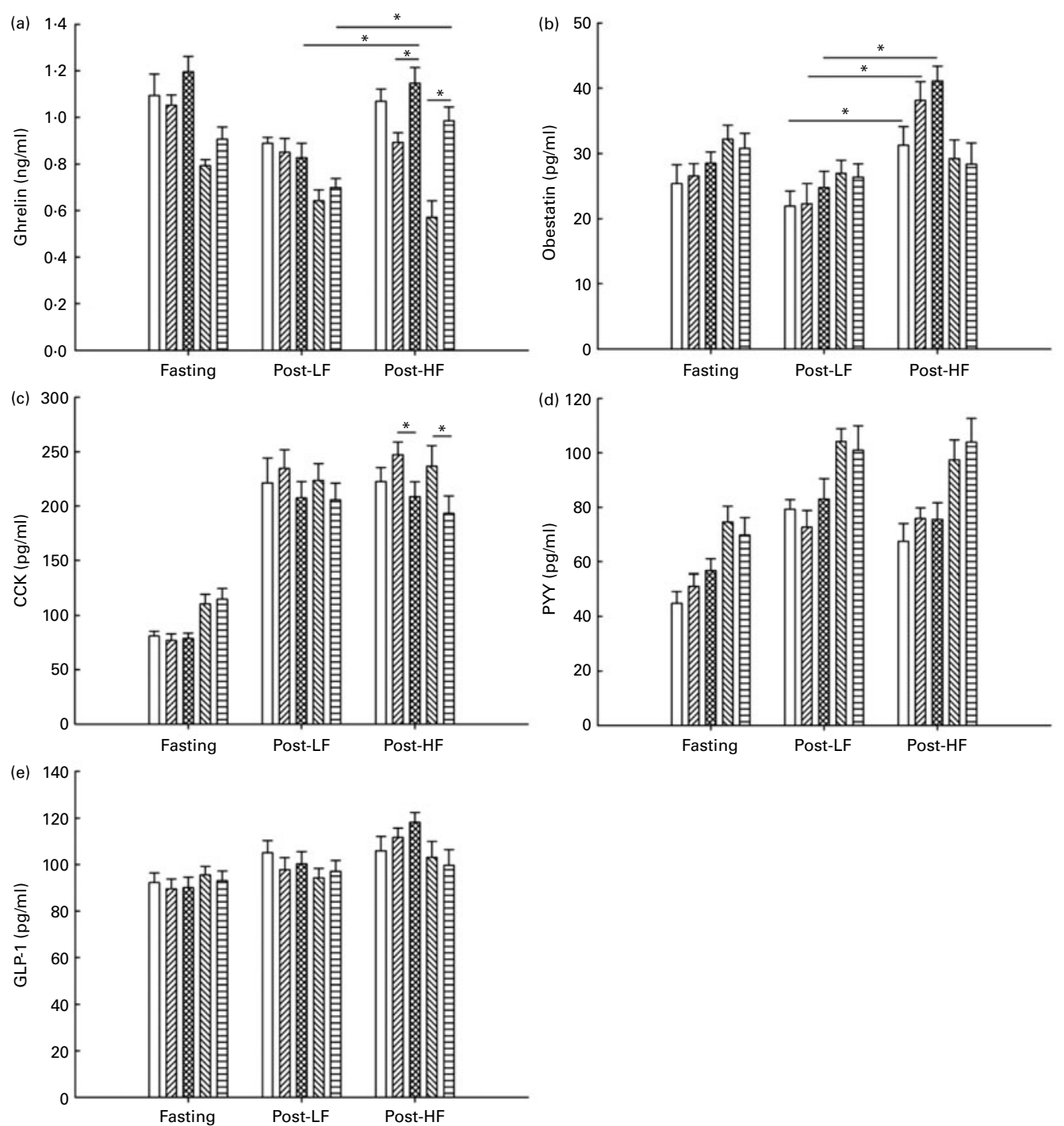

Fig. 4. Plasma ghrelin (a), obestatin (b), cholecystokinin (CCK) (c), peptide tyrosine-tyrosine (PYY) (d) and glucagon-like peptide 1 (GLP-1) (e) concentrations in the control (CON, $\square$ ), diet-resistant rats on high-fat diet (DRHF, 四), diet-resistant rats on low-fat diet (DRLF, 国), diet-induced obese rats on high-fat diet $(\mathrm{DIOHF}, \mathbb{Q})$ and diet-induced obese rats on low-fat diet (DIOLF, 目) rats that were fasted for $12 \mathrm{~h}$ (fasting) and fasted-refed for $1 \mathrm{~h}$ with a LF (post-LF) or a HF (post-HF) diet. Values are means $(n 6-8)$, with standard errors represented by vertical bars. ${ }^{*}$ Mean values were significantly different $(P<0 \cdot 05)$. 
and CON rats. However, the rats that were refed a LF diet did not have significantly higher plasma obestatin concentrations than the fasted rats (Fig. 4(b)).

There were main effects of intervention $(P=0.013)$ and refeeding status $(P<0 \cdot 0001)$ on plasma CCK levels. Switching from a HF diet to a LF diet resulted in a lower plasma CCK concentration $(P=0.013)$. In addition, the rats that were refed with both the HF and LF diets had significantly higher plasma CCK concentrations $(P<0.0001)$ than the fasted rats (Fig. 4(c)).

There were main effects of phenotype $(P<0.0001)$ and refeeding status $(P<0.0001)$ on plasma PYY levels. The DIO rats had significantly higher plasma PYY concentrations than the DR $(P<0.0001)$ and CON $(P<0.0001)$ rats. Moreover, the rats refed with both the $\mathrm{HF}$ and $\mathrm{LF}$ diets had significantly higher plasma PYY concentrations than the fasted rats $(P<0 \cdot 0001 ;$ Fig. $4(d))$.

There was a main effect of refeeding status $(P<0 \cdot 0001)$ on plasma GLP-1 levels. The rats refed with a HF diet had significantly higher plasma GLP-1 concentrations than the fasted rats $(P<0 \cdot 0001)$ and the rats refed with a LF diet $(P=0 \cdot 014 ;$ Fig. $4(\mathrm{e}))$.

Correlations of fasting plasma ghrelin, obestatin, cholecystokinin, peptide tyrosine-tyrosine, glucagon-like peptide-1 concentrations and dietary intervention with body weight

Fasting plasma ghrelin concentrations were negatively correlated with body weight $(r-0.589, P<0.0001, n 8)$. Fasting plasma CCK $(r 0.408, P=0.015, n$ 8) and PYY ( $r$ 0.285, $P=0.047, n$ 8) concentrations were positively correlated with body weight. Multiple regression analysis indicated that only the fasting plasma ghrelin concentration ( $\beta=-0.501, P=0.020, n 8$ ) was an independent predictor of body weight.

\section{Discussion}

The present study is the first to highlight the long- and short-term effects of changing dietary fat content on gut hormones in DIO and DR rats. Switching from a HF diet to a LF diet in DIO and DR rats for 4 weeks can result in less fat mass, a higher fasting and post-HF plasma ghrelin concentration and a lower postprandial plasma CCK concentration. The short-term effects of changing dietary fat content were that post-HF plasma ghrelin concentrations were higher than post-LF concentrations in DRLF and DIOLF rats, and post-HF plasma obestatin concentrations were higher than post-LF concentrations in CON, DRHF and DRLF rats.

Similar to previous observations ${ }^{(16,18)}$, the DIO rats showed significantly higher body weight, energy intake and WAT than the DR and CON rats. Replacing a HF diet with a LF diet significantly reduced energy intake in the DIO, DR and CON rats. However, their energy intake recovered to previous levels after 2 weeks. The DIOLF rats demonstrated a lower body weight than the DIOHF rats during the following 2 weeks after switching from a HF diet to a LF diet. However, this difference disappeared when the analysis was performed with weekly energy intake as a covariate. At the end of this experiment, switching from a HF diet to a LF diet reduced WAT, but not body weight, in the DIO and DR rats. These findings confirmed that diet and genetic background interacted to establish high- (DIO) and low (DR)-body-weight set points, which were then defended against subsequent changes in diet composition and/or energy availability ${ }^{(22)}$. Because of the short-term effects of gut hormones on appetite and the long-term effects on energy homeostasis, gut hormones are speculated to play key roles in the above-mentioned changes in energy intake, body weight and WAT.

In the present study, fasting and post-HF plasma ghrelin concentrations increased after switching from a HF diet to a LF diet in the DIO and DR rats. In line with the previous studies $^{(25,26)}$, the present findings showed that postprandial plasma ghrelin concentrations were suppressed by a LF diet in each group. However, postprandial plasma ghrelin concentrations were suppressed by a HF diet only in the DRHF and DIOHF rats. The CON, DRLF and DIOLF rats, which adapted to a LF diet, had higher postprandial plasma ghrelin concentrations than the DRHF and DIOHF rats, which, in turn, adapted to a HF diet, after being refed an isoenergetic $\mathrm{HF}$ diet. These results support previous reports showing that carbohydrate suppresses ghrelin more potently than fat and protein ${ }^{(27,28)}$. Ghrelin is a physiological meal initiator ${ }^{(26)}$. The high postprandial plasma ghrelin concentration cannot effectively induce satiety. Consequently, the CON, DRLF and DIOLF rats, whose postprandial plasma ghrelin has not been suppressed by a HF diet, will ingest food more than their physiological energy need when they were refed the HF diet. This can partly explain why rats tended to eat more on a HF diet than a LF diet and why switching from a $\mathrm{HF}$ diet to a LF diet reduced the total cumulative energy intake in both the DIO and DR rats.

Furthermore, fasting and post-HF plasma ghrelin concentrations increased after switching from a HF diet to a LF diet in the DIO and DR rats. Therefore, plasma ghrelin concentrations may be affected by body weight and diet composition and may in turn regulate energy intake, body weight and WAT. It has been shown that ghrelin is expressed in human abdominal subcutaneous adipocytes; moreover, ghrelin isoforms appear to mediate fat deposition with the lipogenic effects ${ }^{(4)}$. These data support our finding that plasma ghrelin concentration was associated with WAT.

Zhang et $a l{ }^{(8)}$ reported that obestatin reduces food intake, antagonising the effects of ghrelin. However, some studies have demonstrated that obestatin has no effects on ghrelin-induced hunger or gastric transit ${ }^{(5-7)}$. Furthermore, it remains obscure whether the postprandial 
plasma obestatin level changes in response to a meal. One study has found that plasma obestatin levels do not change in response to a meal in humans ${ }^{(29)}$. In contrast, another study has found that plasma obestatin levels increase significantly in fasting rats compared with rats fed an ad libitum diet ${ }^{(30)}$. In the present study, post-LF plasma obestatin concentrations did not change significantly compared with fasting plasma obestatin concentrations in the rats of each group. However, post-HF plasma obestatin concentrations significantly increased compared with fasting concentrations in the DRLF and DRHF rats, but not in the DIOLF and DIOHF rats. Therefore, it seems that the dietary fat content and phenotype of rats have interaction effects on the postprandial plasma obestatin concentration. Obestatin is speculated to have a potential role in the regulation of energy homeostasis.

The effects of CCK on the gastrointestinal system include inhibiting gastric emptying, food intake, and stimulating gall bladder contraction and pancreatic enzyme secretion $^{(31-33)}$. In line with the previous study ${ }^{(32)}$, plasma CCK concentrations increased one- to twofold after the rats were refed with a HF diet or a LF diet in the present study. The DIO rats had a higher fasting plasma CCK concentration than the DR and CON rats. These results support the previous notion that CCK played a key role in appetite regulation and long-term energy homeostasis. However, no conclusive results were found to prove that an acute switching diet affected the plasma CCK concentration. Although the plasma CCK concentration was related to the rat phenotype, it was not safe to conclude that switching from a HF diet to a LF diet had long-term effects on the plasma CCK concentration.

PYY can delay gastric emptying and reduce gastric secretion $^{(34)}$. In the present study, lowering dietary fat content by switching from a HF diet to a LF diet produced no effects on the fasting and postprandial plasma PYY concentrations. An acute changing dietary fat content seemingly had no effect on the plasma PYY concentration. These results were different from the report which shows that lowering dietary fat content can lower plasma total PYY in the DIO rats ${ }^{(23)}$. Differences in rodents, experimental design and the detected isoform of PYY may be the reasons for this divergence.

GLP-1 is a potent incretin, which suppresses mealinduced gastric acid and pancreatic juice secretion and delays gastric emptying ${ }^{(35,36)}$. It is believed to act as a satiety signal and possibly to play a key role in long-term energy homeostasis. In the present study, post-HF plasma GLP-1 concentrations increased significantly in the DRHF and DRLF rats but not in the CON, DIOHF and DIOLF rats. It is considered that GLP-1 has inhibitory effects on food intake ${ }^{(37)}$. Therefore, the sensitive response of GLP-1 to a HF diet in the DR rats may explain why they ingested less energy and had lower body weights and WAT than the DIO rats, to a certain degree. However, the positive longand short-term effects of changing dietary fat content on the plasma GLP-1 concentrations were not observed in the present study.

Several limitations of the present study should be explained. First, we only detected postprandial plasma gut hormone concentrations at one time point. In the present study, the primary aims were to research the long- and short-term effects of changing dietary fat content on gut hormones. The fasting, post-HF and post-LF plasma gut hormone concentrations can reflect these effects to some extent. In future studies, the time profile of gut hormone secretion will be obtained to determine the effects of changing dietary fat content on gut hormones in detail. Second, the causality between energy intake, body weight, WAT changes and plasma gut hormone concentrations cannot be confirmed in the present study. The agonist and antagonist of gut hormones may be helpful to clarify this problem. Third, although changing dietary fat content produced many effects on gut hormones, simply attributing these effects to dietary fat content per se is inappropriate. Energy intake, palatability of foods, stress, sex and activity, in addition to dietary composition, can affect gut hormones. Future studies should focus on the mechanisms underlying these effects. Fourth, only the main plasma isoform, but not all isoforms, of some gut hormones was detected. Fifth, no data on energy expenditure, activity level and resting energy levels, which may explain the different 'phenotype', were observed. However, some published data ${ }^{(16,17,22)}$ can be referred to, which can help us to grasp the characteristics of HF DIO rats.

In conclusion, replacing a HF diet with a LF diet for 4 weeks resulted in lower WAT and regulated the plasma ghrelin and CCK concentrations in DIO and DR rats. Acute changing dietary fat content had effects on plasma ghrelin and obestatin concentrations. Moreover, it has been shown that gut hormones play key roles in energy homeostasis. Thus, we demonstrate that changing dietary fat content appears to play long- and short-term roles in the gut hormone profile, which may consequently influence energy intake and fat mass.

\section{Acknowledgements}

The present study was funded by the Natural Science Foundation of China 30872108. The authors' responsibilities were as follows: J. L. and S. W. designed the research; J. L., N. Z., Z. L., R. L. and C. L. conducted the research; J. L. analysed the data; J. L. wrote the manuscript; J. L. and S. W. had primary responsibility for the final content. All authors read and approved the final manuscript. There are no conflicts of interest.

\section{References}

1. Murphy KG \& Bloom SR (2006) Gut hormones and the regulation of energy homeostasis. Nature 444, 854-859. 
2. Tschop M, Smiley DL \& Heiman ML (2000) Ghrelin induces adiposity in rodents. Nature $\mathbf{4 0 7}, \mathbf{9 0 8 - 9 1 3}$

3. Wren AM, Small CJ, Ward HL, et al. (2000) The novel hypothalamic peptide ghrelin stimulates food intake and growth hormone secretion. Endocrinology 141, 4325-4328.

4. Kos K, Harte AL, O'Hare PJ, et al. (2009) Ghrelin and the differential regulation of des-acyl (DSG) and oct-anoyl ghrelin (OTG) in human adipose tissue (AT). Clin Endocrinol (Oxf) 70, 383-389.

5. Gourcerol G, Coskun T, Craft LS, et al. (2007) Preproghrelinderived peptide, obestatin, fails to influence food intake in lean or obese rodents. Obesity (Silver Spring) 15, 2643-2652.

6. Nogueiras R, Pfluger P, Tovar S, et al. (2007) Effects of obestatin on energy balance and growth hormone secretion in rodents. Endocrinology 148, 21-26.

7. Seoane LM, Al-Massadi O, Pazos Y, et al. (2006) Central obestatin administration does not modify either spontaneous or ghrelin-induced food intake in rats. J Endocrinol Invest 29, RC13-RC15.

8. Zhang JV, Ren PG, Avsian-Kretchmer O, et al. (2005) Obestatin, a peptide encoded by the ghrelin gene, opposes ghrelin's effects on food intake. Science 310, 996-999.

9. Abbott CR, Monteiro M, Small CJ, et al. (2005) The in-hibitory effects of peripheral administration of peptide YY(3-36) and glucagon-like peptide- 1 on food intake are attenuated by ablation of the vagal-brainstem-hypothalamic pathway. Brain Res 1044, 127-131.

10. Acuna-Goycolea C \& van den Pol AN (2005) Peptide YY(3-36) inhibits both anorexigenic proopiomelanocortin and orexigenic neuropeptide Y neurons: implications for hypothalamic regulation of energy homeostasis. $J$ Neurosci 25, 10510-10519.

11. Batterham RL, Cowley MA, Small CJ, et al. (2002) Gut hormone PYY(3-36) physiologically inhibits food intake. Nature 418, 650-654.

12. Gibbs J, Young RC \& Smith GP (1973) Cholecystokinin elicits satiety in rats with open gastric fistulas. Nature $\mathbf{2 4 5}$ 323-325.

13. Adams SH, Lei C, Jodka CM, et al. (2006) PYY[3-36] administration decreases the respiratory quotient and reduces adiposity in diet-induced obese mice. J Nutr 136, 195-201.

14. Chelikani PK, Haver AC \& Reidelberger RD (2007) Intermittent intraperitoneal infusion of peptide $\mathrm{YY}(3-36)$ reduces daily food intake and adiposity in obese rats. Am J Physiol Regul Integr Comp Physiol 293, R39-R46.

15. Boey D, Lin S, Enriquez RF, et al. (2008) PYY transgenic mice are protected against diet-induced and genetic obesity. Neuropeptides 42, 19-30.

16. Levin BE \& Keesey RE (1998) Defense of differing body weight set points in diet-induced obese and resistant rats. Am J Physiol 274, R412-R419.

17. Levin BE, Triscari J \& Sullivan AC (1983) Relationship between sympathetic activity and diet-induced obesity in two rat strains. Am J Physiol 245, R364-R371.

18. Zhao D, Wang SR, Ma WW, et al. (2008) Alpha1-macroglobulin: a potential obesity-related factor in serum. Med Sci Monit 14, BR57-BR61.

19. Buettner R, Scholmerich J \& Bollheimer LC (2007) High-fat diets: modeling the metabolic disorders of human obesity in rodents. Obesity (Silver Spring) 15, 798-808.

20. Speakman J, Hambly C, Mitchell S, et al. (2007) Animal models of obesity. Obes Rev 8, Suppl. 1, 55-61.
21. Furnes MW, Zhao CM \& Chen D (2009) Development of obesity is associated with increased calories per meal rather than per day. A study of high-fat diet-induced obesity in young rats. Obes Surg 19, 1430-1438.

22. Levin BE, Triscari J, Hogan S, et al. (1987) Resistance to dietinduced obesity: food intake, pancreatic sympathetic tone, and insulin. Am J Physiol 252, R471-R478.

23. Huang XF, Yu Y, Li Y, et al. (2008) Ventromedial hypothalamic NPY Y2 receptor in the maintenance of body weight in diet-induced obesity in mice. Neurochem Res 33, $1881-1888$

24. Aziz AA, Kenney LS, Goulet B, et al. (2009) Dietary starch type affects body weight and glycemic control in freely fed but not energy-restricted obese rats. J Nutr 139, 1881-1889.

25. Cummings DE, Weigle DS, Frayo RS, et al. (2002) Plasma ghrelin levels after diet-induced weight loss or gastric bypass surgery. $N$ Engl J Med 346, 1623-1630.

26. Cummings DE, Purnell JQ, Frayo RS, et al. (2001) A preprandial rise in plasma ghrelin levels suggests a role in meal initiation in humans. Diabetes 50, 1714-1719.

27. Erdmann J, Topsch R, Lippl F, et al. (2004) Postprandial response of plasma ghrelin levels to various test meals in relation to food intake, plasma insulin, and glucose. J Clin Endocrinol Metab 89, 3048-3054

28. Monteleone P, Bencivenga R, Longobardi N, et al. (2003) Differential responses of circulating ghrelin to high-fat or high-carbohydrate meal in healthy women. J Clin Endocrinol Metab 88, 5510-5514.

29. Huda MS, Durham BH, Wong SP, et al. (2008) Plasma obestatin levels are lower in obese and post-gastrectomy subjects, but do not change in response to a meal. Int J Obes (Lond) 32, 129-135.

30. Guo ZF, Ren AJ, Zheng X, et al. (2008) Different responses of circulating ghrelin, obestatin levels to fasting, re-feeding and different food compositions, and their local expressions in rats. Peptides 29, 1247-1254

31. Dockray G (2004) Gut endocrine secretions and their relevance to satiety. Curr Opin Pharmacol 4, 557-560.

32. Liddle RA, Goldfine ID, Rosen MS, et al. (1985) Cholecystokinin bioactivity in human plasma. Molecular forms, responses to feeding, and relationship to gallbladder contraction. J Clin Invest 75, 1144-1152.

33. Moran TH \& McHugh PR (1982) Cholecystokinin suppresses food intake by inhibiting gastric emptying. Am J Physiol 242, R491-R497.

34. Taylor IL (1993) Role of peptide YY in the endocrine control of digestion. J Dairy Sci 76, 2094-2101.

35. Nauck MA, Niedereichholz U, Ettler R, et al. (1997) Glucagon-like peptide 1 inhibition of gastric emptying outweighs its insulinotropic effects in healthy humans. Am J Physiol 273, E981-E988.

36. Wettergren A, Schjoldager B, Mortensen PE, et al. (1993) Truncated GLP-1 (proglucagon 78-107-amide) inhibits gastric and pancreatic functions in man. Dig Dis Sci 38, 665-673.

37. Flint A, Raben A, Ersboll AK, et al. (2001) The effect of physiological levels of glucagon-like peptide-1 on appetite, gastric emptying, energy and substrate metabolism in obesity. Int J Obes Relat Metab Disord 25, 781-792.

38. Reeves PG, Nielsen FH \& Fahey GC Jr (1993) AIN-93 purified diets for laboratory rodents: final report of the American Institute of Nutrition ad hoc writing committee on the reformulation of the AIN-76A rodent diet. J Nutr 123, 1939-1951. 